\title{
Strain hardening behaviors and strain rate sensitivity of gradient-grained Fe under compression over a wide range of strain rates
}

\author{
Fuping Yuan*, Ping Chen, Yanpeng Feng, Ping Jiang, Xiaolei Wu \\ State Key Laboratory of Nonlinear Mechanics, Institute of Mechanics, Chinese Academy of Science, No. 15, North 4th Ring, West Road, \\ Beijing 100190, China
}

\section{A R T I C L E I N F O}

\section{Article history:}

Received 10 September 2015

Revised 18 November 2015

Available online 12 January 2016

\section{Keywords:}

Dynamic loading

Strain hardening behaviors

Strain rate sensitivity

Gradient-grained structure

Coarse-grained structure

\begin{abstract}
A B S T R A C T
In the present work, gradient-grained Fe was synthesized by means of surface mechanical grinding treatment, and then the compression behaviors of the coarse-grained Fe and the gradient-grained Fe were investigated under both quasi-static and dynamic loading conditions over a wide range of strain rates (from $5 \times 10^{-4}$ to $10^{4} \mathrm{~s}^{-1}$ ). After surface mechanical grinding treatment, equiaxed ultrafine grains, elongated lamellar ultrafine grains, full-developed sub-grains with dense dislocations walls, non-fully-developed dislocation cells, and deformed coarse grains are sequentially observed along the depth from the treated surface. The grain/cell size increases while the measured micro-hardness decreases along the depth for the gradient-grained Fe. The gradient-grained structure shows apparent strain hardening behaviors at all strain rates up to $10^{4} \mathrm{~s}^{-1}$ although the strain hardening exponent $(n)$ for the gradient-grained Fe is smaller than that of the coarse-grained Fe at the same strain rate. This apparent hardening behavior is attributed to the hardening from both the coarse-grained center and the surface gradient layers when the strain localization trend for the ultrafine-grained surface layers is suppressed by the coarse-grained center. The extra hardening might be due to the back stress hardening associated with the constraint and mechanical incompatibility between different layers in the gradient-grained structure. The dynamic strain rate sensitivity of the gradient-grained $\mathrm{Fe}$ is observed to be slightly larger than that of the coarse-grained Fe, which is controversial to the general observation that strain rate sensitivity should decrease with reduction of grain size for BCC metals. The geometrically necessary dislocations associated with the back stress hardening and the grain size gradient result in additional increase in dislocation density, which may be the reason for the enhanced dynamic strain rate sensitivity in the gradient-grained Fe even it has smaller average grain size compared to the coarse-grained Fe. The present results should provide insights for the applications of gradient-grained structure under dynamic conditions.
\end{abstract}

(c) 2016 Elsevier Ltd. All rights reserved.

\section{Introduction}

In recent years, bulk ultrafine-grained (UFG) and nanograined (NG) metals, which are commonly produced by

\footnotetext{
* Corresponding author. Tel.: +86 108254 4409;

fax: +86108254 3977/+86108254 4409 .

E-mail address: fpyuan@lnm.imech.ac.cn (F. Yuan).
}

means of severe plastic deformation (SPD) techniques, have drawn increasing interests due to their ultra-high strength (Meyers et al., 2006; Dao et al., 2007). However, they show limited ductility compared to their coarsegrained (CG) counterparts due to the reduced strain hardening rate (Valiev, 2004; Zhu and Liao, 2004). Stronger and tougher metals and alloys are always desired for the structural applications in modern industry, and such 
demands have been realized recently by several novel strategies through tailoring microstructures at nano-scale, such as, bimodal grain size design, nano-precipitate dispersion, pre-existing growth nano-twins, and gradient nanograined (GNG) structure (Wang et al., 2002; Liddicoat et al., 2010; Liu et al., 2013; Lu et al., 2009; Suresh, 2011; Fang et al., 2011; Wu et al., 2014a; Wei et al., 2014).

The GNG/CG hierarchical architecture, consisting of the CG core sandwiched by two GNG surface layers, can produce excellent synergy of strength and ductility (Lu and Lu, 2004; Chen et al., 2008; Fang et al., 2011; Wu et al., 2014a; Wei et al., 2014). Strain localization can be suppressed in the NG Cu film when confined by a CG core with a gradient grain size transition due to a mechanicallydriven and strain-induced growth of nano-grains (Fang et al., 2011). Our previous work showed that large plastic strain could also be achieved in NG surface layer of $\mathrm{Fe}$ without apparent grain growth when confined by the CG core (Wu et al., 2014a). These evidences suggested that the variant trends for strain instability between different layers in GNG/CG architecture usually induce stress state change to suppress the strain localization of the NG surface layers. The mechanical incompatibility should also produce back stress hardening associated with a strain process providing long-range interactions with mobile dislocations (such as geometrically necessary dislocations, GNDs) (Feaugas, 1999; Elliot et al., 2004). Moreover, the constraint and mechanical incompatibility between different layers should also induce the strain gradient, which produces the extra hardening and the synergic strengthening due to the grain size gradient (Gao et al., 1999; Wu et al., 2014a,b).

The strain hardening behaviors and the observed flow stresses for resisting plastic deformation of metals and alloys also highly depend on the rate associated with loading (Zener and Hollomon, 1944; Subhash, 1995; Nemat-Nasser and Guo, 2003; Song et al., 2007; Mishra et al., 2008). Previous research have indicated that the grain size (especially down to the UFG or NG regimes) has strong influences on the strain rate dependent plastic deformation, such as the strain rate sensitivity (SRS) and the rate-controlling deformation mechanisms (Wei et al., 2004, 2006a,b; Wei, 2007; Mishra et al., 2008; Suo et al., 2011, 2013a; Yu et al., 2015). The effect of grain size on SRS has been observed to be strongly dependent on the lattice structures (Wei, 2007). The SRS of FCC metal increases with the reduction of grain size, while the SRS of BCC metals shows opposite behaviors. To date, numerous efforts have been undertaken to investigate the tensile properties and the strain harden- ing behaviors of nanostructured metals under quasi-static conditions (Malow and Koch, 1998; Tsuji et al., 2002; Han et al., 2003; Conrad, 2004; Han et al., 2004; Valiev, 2004; Giga et al., 2006; Meyers et al., 2006; Hazra et al., 2011). There also have been a number of investigations on the effect of high strain rate on the mechanical behaviors of UFG or NG metals (Jia et al., 1999; Wei et al., 2004, 2006a; Mishra et al., 2008; Suo et al., 2011, 2013a; Yu et al., 2015). However, most of reported research work on dynamic deformation of nanostructured metals was based on UFG or NG metals with relatively homogeneous grain size. There is clearly a lack of understanding of the effect of strain rate on the plastic deformation mechanisms for gradientgrained structure. In this regard, strain hardening behaviors and strain rate sensitivity of gradient-grained Fe under compression are systematically examined over a wide range of strain rates, and the corresponding plastic deformation behaviors are also compared with the CG counterpart.

\section{Experimental procedures}

The commercial pure iron used in the present study was received in the form of rods of $10 \mathrm{~mm}$ in diameter. The as-received materials have a composition, in weight percentage, of $0.008 \mathrm{C}, 0.02 \mathrm{Si}, 0.08 \mathrm{Mn}, 0.009 \mathrm{P}, 0.008 \mathrm{~S}$, $0.01 \mathrm{Cr}, 0.01 \mathrm{Ni}, 0.01 \mathrm{Cu} 0.06 \mathrm{Al}$, and the balance of Fe. The as-received rods were first annealed at $900{ }^{\circ} \mathrm{C}$ for $2 \mathrm{~h}$ to obtain a CG polycrystalline structure with a single ferrite phase. After annealing, the rods were processed by surface mechanical grinding treatment (SMGT) to synthesize a gradient-grained structure. SMGT is a method adopted from machining at high strain rates (up to $10^{3}-10^{6} \mathrm{~s}^{-1}$ ). As shown in Fig. 1(a), a cylindrical WC/Co tool tip repetitively slides at a velocity of $v_{1}$ along the gauge section of the dog-bone shaped sample (with a diameter of 10 $\mathrm{mm}$ for the ends and a diameter of $3 \mathrm{~mm}$ for the gauge section), which rotates at a velocity of $v_{2}$ with respect to the tool tip. A high-rate plastic shear deformation zone with high strain gradient is induced underneath the tip and is applied to the top surface layer of the sample when a preset penetration depth (20 $\mu \mathrm{m}$ for each time) into the sample is applied to the tool tip. The magnitude of induced plastic strain and the depth of the deformed layer are closely related to the tip diameter $(3 \mathrm{~mm})$ and the total penetration depth $(180 \mu \mathrm{m})$, while the shear deformation rate is determined by the velocities of $v_{1}$ (3 $\mathrm{mm} / \mathrm{s}$ ) and $v_{2}(600 \mathrm{rpm})$. Effective cooling system with liquid nitrogen is applied to the processed material and

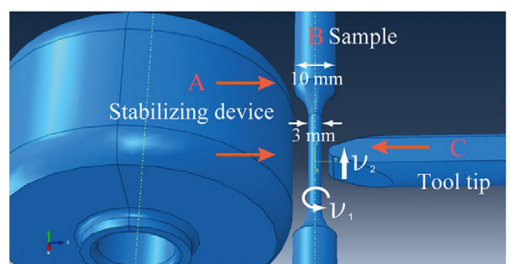

a
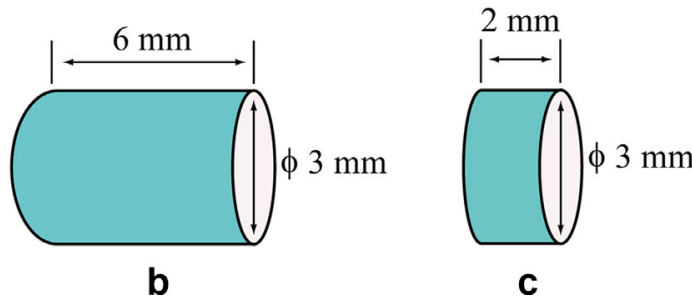

Fig. 1. (a) Schematic of SMGT; (b) dimensions of the specimens for quasi-static compression experiments; (c) dimensions of the specimens for dynamic compression experiments. 
the tool tip during SMGT in order to refine the started coarse grains into nanoscale regime.

The microstructure after SMGT was examined by optical microscope (OM), electron backscattered diffraction (EBSD), transmission electron microscope (TEM) and micro-hardness measurements. The sample surfaces for OM were polished to 2000 grit and finally polished with $0.25 \mu \mathrm{m}$ diamond paste. This was followed by etching with $5 \%$ Nital. The cross-sectional surfaces of the SMGT samples for EBSD were first polished to 2000 grit and finally polished with $0.25 \mu \mathrm{m}$ diamond paste, and then were electropolished with $5 \%$ perchloric acid at $37 \mathrm{~V}$ voltage and -20 ${ }^{\circ} \mathrm{C}$ for $10-15 \mathrm{~s}$ to reveal the microstructure. Using a field emission gun and the low accelerating voltage, the spatial resolution of EBSD can be significantly improved, making it possible to successfully explore the microstructure even in severely deformed state (Chen et al., 2011a,b; Sun et al., 2014; Yuan et al., 2015). The cross-sectional disks for TEM were cut with a thickness of $500 \mu \mathrm{m}$ and polished down to $30 \mu \mathrm{m}$ using 2000 grid SiC papers. Final thinning to electron transparency was achieved by ion milling at various depths from the treated surface. Micro-hardness measurements were also made on the cross-sectional surface of the SMGT samples before and after dynamic compression tests using a Vickers diamond indenter at a load of 10 $\mathrm{g}$ for $10 \mathrm{~s}$ dwell time. The light load is especially suitable for measurements along the depth for the gradient structure, in which the indentation size is $\sim 7 \mu \mathrm{m}$ and the vertical spacing for indentations is $\sim 22 \mu \mathrm{m}$ (the real spacing for indentations is larger since sawtooth pattern was used) at the top surface layers.

All SMGT samples for quasi-static and dynamic compression testing were machined from the gauge section of the processed samples by wire saw with loading direction parallel to the axis of rods. The dimensions of samples for quasi-static and dynamic compression testing are given in Fig. 1(b) and (c), respectively. Quasi-static uniaxial compression tests were carried out using a MTS 810 testing machine with a maximum load capacity of $25 \mathrm{kN}$ at a strain rate of $0.0005 \mathrm{~s}^{-1}$. An extensometer was used to measure the strain during the compression loading.

Dynamic compression tests were performed using Hopkinson-bar techniques. Details of the Hopkinson-bar technique and the data analysis for true stress, true strain and true strain rate can be found elsewhere (Subhash et al., 1997; Song et al., 2007, Sunny et al., 2009). Grease was used between the bars and the specimens to ensure low frictions. A pulse shaper was also placed between the striker and the input bar to control the shape of the incident pulse, and thus promote equilibrium conditions within the specimens. Semiconductor strain gages were used on both the incident and transmitted bars to obtain a very high signal-to-noise ratio for the strain measurements on both bars. Then, the recorded reflected and transmitted signals can be used to calculate the engineering stress, the engineering strain and the engineering strain rate

$\sigma_{s}=E\left(\frac{A}{A_{s}}\right) \varepsilon_{T}$ $\varepsilon_{s}=-\frac{2 C_{0}}{l_{s}} \int_{0}^{t} \varepsilon_{R} d \tau$

$\dot{\varepsilon}_{s}=-\frac{2 C_{0}}{l_{S}} \varepsilon_{R}$

where $\varepsilon_{T}$ and $\varepsilon_{R}$ are the transmitted and reflected strain pulses for the input and output bars, respectively; $C_{0}, E$ and $A$ are the longitudinal elastic wave velocity, Young's modulus and the cross-sectional area of the loading bars, respectively; $l_{s}$ and $A_{s}$ are the length and the cross-sectional area of the specimens, respectively. Finally, the true stresstrue strain curves can be obtained from these engineering stress-engineering strain curves. For the dynamic compression tests, the strain rates were controlled at $\sim 1500$, $\sim 6000, \sim 10,000 \mathrm{~s}^{-1}$ depending on the striker bar velocity. At least two experiments for each strain rate were performed.

\section{Experimental results and discussions}

The as-annealed structure and the gradient-grained structure after SMGT by OM are shown in Fig. 2(a) and (b), respectively. The grain size for the as-annealed sample is varied from $10 \mu \mathrm{m}$ to $100 \mu \mathrm{m}$, and the average grain size is about $50 \mu \mathrm{m}$. While the SMGT sample shows apparent deformed band structure at the top surface of about 150 $\mu \mathrm{m}$, the treated surface is on the right side. The flow direction of the band structure is consistent with the shear deformation direction.

The deformed microstructures at the areas close to the treated surface (up to $70 \mu \mathrm{m}$ from the treated surface) were characterized using EBSD. Fig. 3(a) shows the image quality map by EBSD, in which blue color $\left(>15^{\circ}\right)$ represents high-angle boundaries, green color $\left(5-15^{\circ}\right)$ and red color $\left(2-5^{\circ}\right)$ represent low-angle boundaries. Fig. 3(b) shows the EBSD orientation map. The orientation map shown in the present study is based on the inverse pole figure coloring scheme relative to the direction of cross section. The color codes follow the inserted triangle in Fig. 3(b), in which red, green and blue colors represent the grains having [0 $\left.\begin{array}{ll}0 & 1\end{array}\right]$, [ [ $\left.\begin{array}{lll}1 & 0 & 1\end{array}\right]$ and [ [ $\left.\begin{array}{lll}1 & 1 & 1\end{array}\right]$ directions parallel to the direction of cross section, respectively. The misorientation angle distribution along the dash line of Fig. 3(b) is shown in Fig. 3(c), in which the distance of scanning is from the left to the right. As observed, the outermost layer of $\sim 5 \mu \mathrm{m}$ thickness are nearly equiaxed ultrafine grains with highangle boundaries, while the sub-surface layer of $\sim 20 \mu \mathrm{m}$ thickness are elongated lamellar ultrafine grains with highangle boundaries. At the depths of 25-50 $\mu \mathrm{m}$, the microstructure exhibits strong $\left\langle\begin{array}{llll}1 & 1 & 0\end{array}\right\rangle$ texture, which may be due to $\left\langle\begin{array}{lll}1 & 1 & 0\end{array}\right\rangle$ texture trend by cold work for BCC metals. The misorientation angles for various boundaries decrease with increasing depth from the treated surface due to the strain gradients induced by SMGT, as shown in Fig. 3(c).

The plastic strain deformation by SMGT can induce high strain gradients at the surface layers of the samples. Thus the surface layers of the samples after SMGT also have characteristic of high gradient microstructures (Li et al., 2008; Fang et al., 2011; Wu et al., 2014a). Fig. 4(a)-(f) 


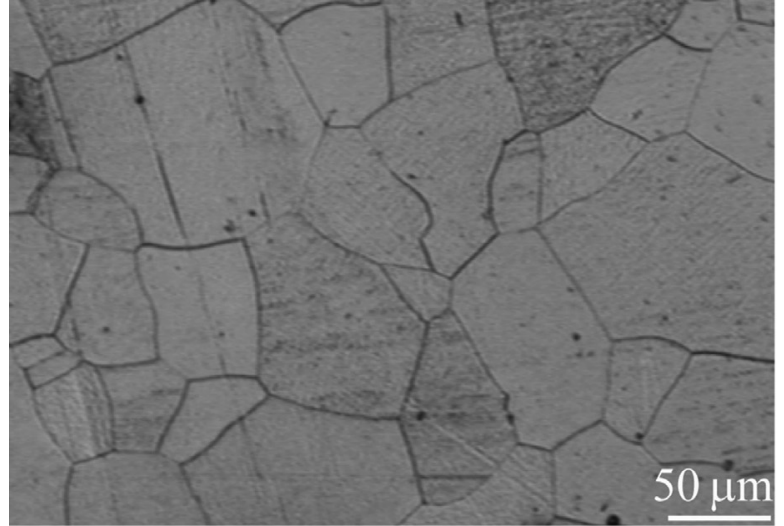

a

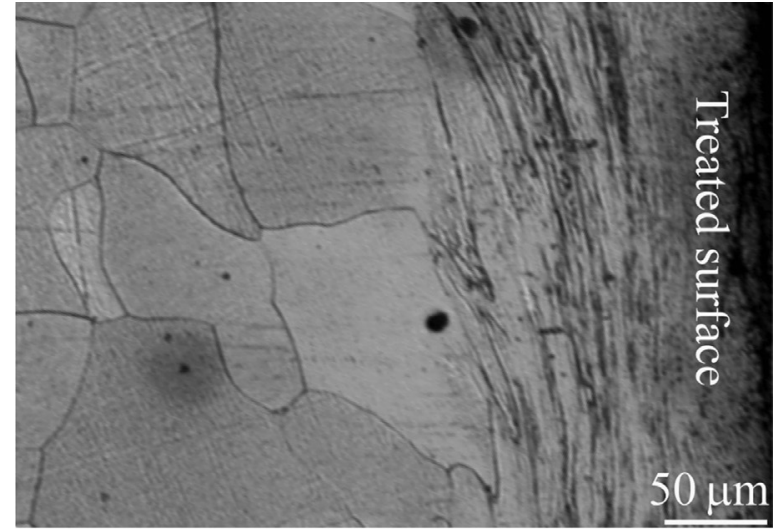

b

Fig. 2. (a) $\mathrm{OM}$ of the as-annealed Fe; (b) $\mathrm{OM}$ of the gradient-grained Fe.

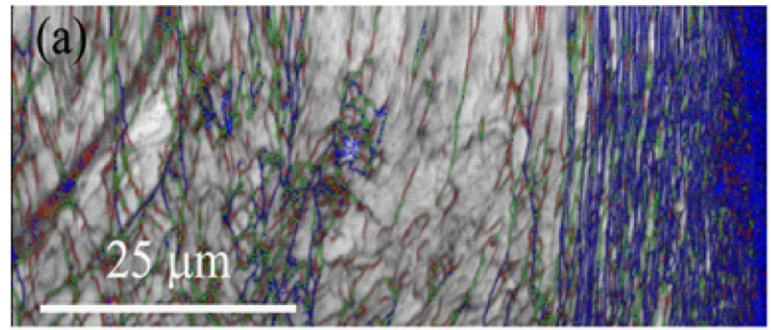
Boundary misorientation
$2^{\circ} \sim 5^{\circ}$
$5^{\circ} \sim 15^{\circ}$
$15^{\circ} \sim 180^{\circ}$

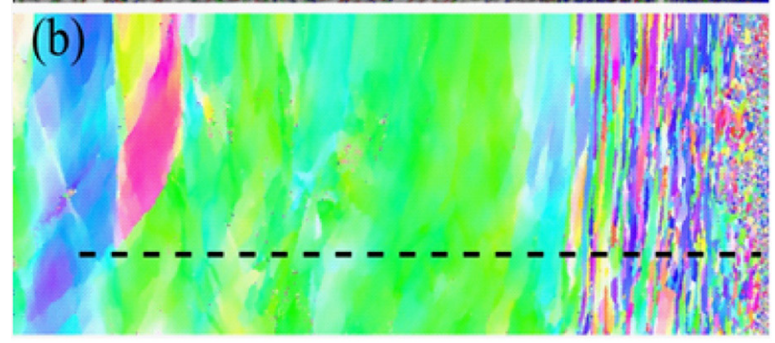

\section{Ferritte

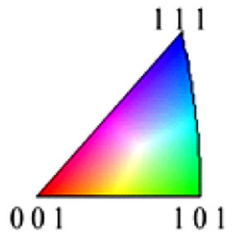

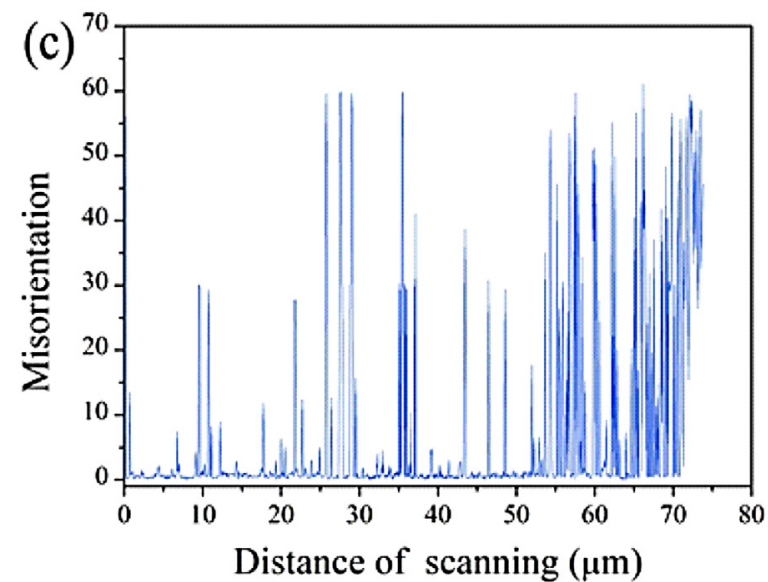

Fig. 3. (a) EBSD micrograph with misorientation angles for the gradient-grained Fe; (b) EBSD orientation map for the gradient-grained Fe; (c) misorientation angle distribution along the dash line in Fig. 3(b). (For interpretation of the references to color in this figure legend, the reader is referred to the web version of this article.) 

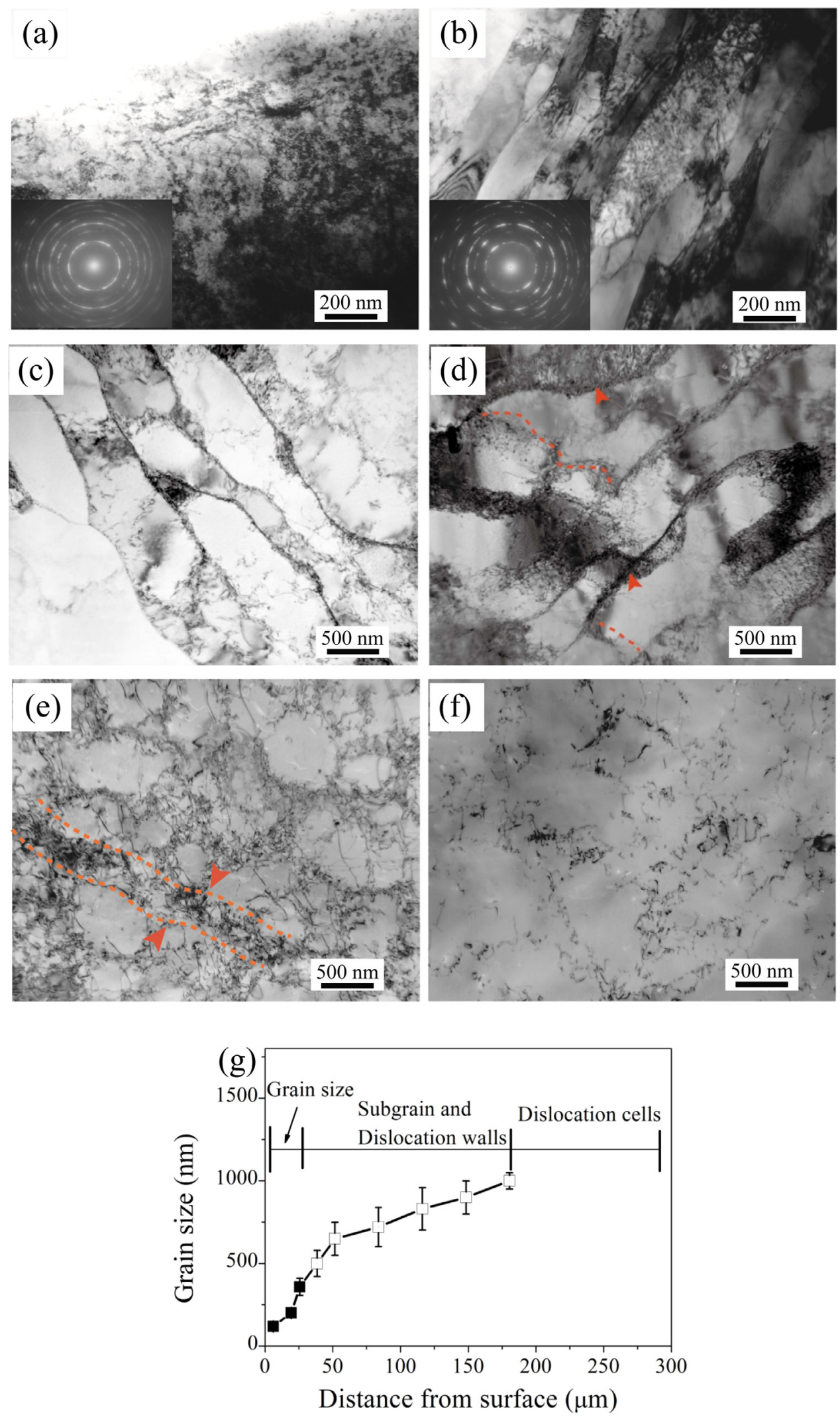

Fig. 4. Bright-field TEM micrographs at various depths from the treated surface for the gradient-grained Fe: (a) Close to the surface area; (b) $\sim 20 \mu \mathrm{m}$; (c) $\sim 80 \mu \mathrm{m}$; (d) $\sim 130 \mu \mathrm{m}$; (e) $\sim 180 \mu \mathrm{m}$; (f) $\sim 230 \mu \mathrm{m}$. (g) Variation of average grain/cell size along the depth from the treated surface. (For interpretation of the references to color in this figure legend, the reader is referred to the web version of this article.) 

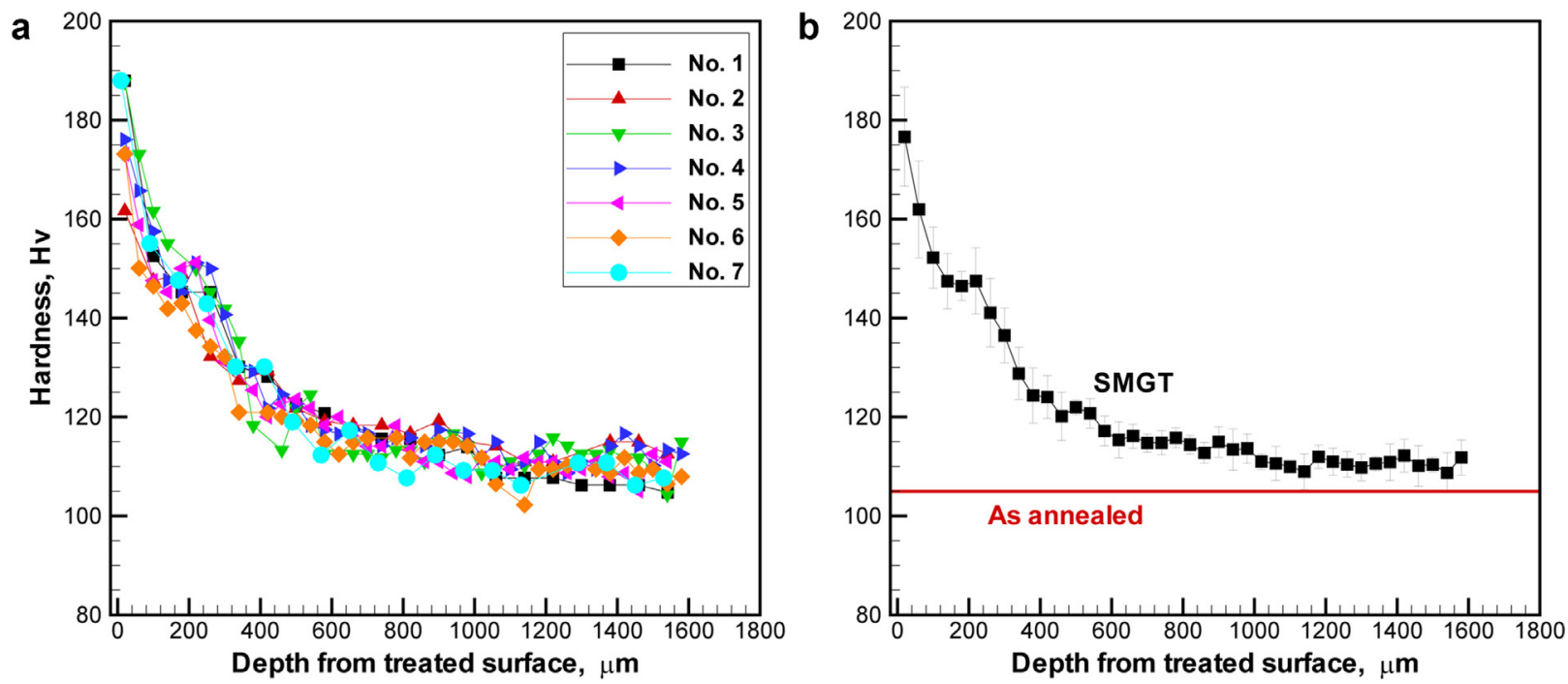

Fig. 5. Vickers micro-hardness distributions along the depth from the treated surface for SMGT Fe: (a) seven groups of measurements; (b) average hardness.

shows bright-field TEM micrographs at various depths from the treated surface for the gradient-grained Fe. The corresponding selected area diffraction (SAD) patterns for Fig. 4(a) and (b) are also shown in the insets. The outermost layer of the gradient-grained Fe (Fig. 4(a)) are nearly equiaxed ultrafine grains with a mean size of $\sim 100 \mathrm{~nm}$, and the SAD patterns show random orientations for all grains. At the depth of $20 \mu \mathrm{m}$ (Fig. 4(b)), the microstructure shows elongated lamellar ultrafine grains with a mean lamellar thickness of $\sim 200 \mathrm{~nm}$, and high density dislocations are also observed inside the lamellar grains. At the depths of 80 and $130 \mu \mathrm{m}$ (Fig. 4(c) and (d)), the initial coarse grains are divided into sub-grains with a mean size of $700-800 \mathrm{~nm}$ by dense dislocation walls (DDWs) or fully-developed dislocation cells. High density of dislocations and dislocation tangles are also observed in the sub-grains. In Fig. 4(d), the boundaries of dislocation cells are marked by the red dash lines, and DDWs are marked by the red arrows. At the depths of 180 and $230 \mu \mathrm{m}$ (Fig. 4(e) and (f)), the deformed microstructures are the non-fully-developed dislocation cells and dislocation tangles are mainly concentrated at the boundaries of cells. In Fig. 4(f), the areas with high density dislocation tangles are marked by the red dash lines and the red arrow. Based on observations from TEM and the statistical analysis, the variation of average grain/cell size along the depth from the treated surface is plotted in Fig. $4(\mathrm{~g})$. Up to the depth of $30 \mu \mathrm{m}$, equiaxed ultrafine grains or elongated lamellar ultrafine grains are observed. Between the depths of $30 \mu \mathrm{m}$ and $175 \mu \mathrm{m}$, sub-grains and full-developed dislocations walls are observed. Between the depths of $175 \mu \mathrm{m}$ and $290 \mu \mathrm{m}$, non-fully-developed dislocation cells are observed. With further increasing depths, the deformed coarse grains are observed since the dislocation density further decreases. As we know, Fe is a typical BCC metal with a high stacking faults energy (SFE) of about $200 \mathrm{~mJ} / \mathrm{m}^{2}$. Thus, the grain refinement process during SMGT can be summarized as the following four steps and mechanisms: (i) increasing of dislocation density in the coarse grains; (ii) development of DDWs or dislocation cells; (iii) transformation of DDWs and dislocation cells into sub-grains with small misorientations; (iv) evolution of sub-grains to highly-misoriented grains with equiaxed shape or lamellar shape.

Fig. 5 shows Vickers micro-hardness distributions along the depth from the treated surface for the gradient-grained Fe. Seven groups of measurements along the depths were made, and the average value was taken for reducing the physical errors. The standard deviations for seven group measurements are plotted as error bars in Fig. 5(b). The hardness for the as-annealed Fe is also plotted as a straight line in Fig. 5(b) for comparison. As shown, the microhardness decreases from $180 \mathrm{Hv}$ at the top surface ( $10 \mu \mathrm{m}$ from the treated surface) to $110 \mathrm{Hv}$ at the center (slightly higher than the $105 \mathrm{Hv}$ for un-deformed coarse grains) for SMGT Fe. The continuously decreasing hardness along the depth is consistent with the increasing grain/cell size, as shown in Fig. 4(g).

The mechanical responses of the as-annealed Fe and the gradient-grained Fe under quasi-static compression (strain rate of $0.0005 \mathrm{~s}^{-1}$ ) are presented here. Fig. 6(a) shows the engineering stress-strain curves, Fig. 6(b) shows the true stress-strain curves, while Fig. 6(c) shows the hardening rate $\left(\Theta=\frac{d \sigma}{d \varepsilon}\right)$ curves as a function of the true strain. The yield strength of the gradient-grained $\mathrm{Fe}$ is estimated to be $\sim 300 \mathrm{MPa}$, which is almost two times of that for the as-annealed Fe. Although both the as-annealed Fe and the gradient-grained Fe show apparent strain hardening behaviors up to strain of $60 \%$, the strain hardening ability of the gradient-grained $\mathrm{Fe}$ is observed to be smaller compared to that of the as-annealed Fe. As shown in Fig. 6(b), the difference of the flow stress between the as-annealed Fe and the gradient-grained $\mathrm{Fe}$ decreases with increasing strain. The as-annealed Fe also shows a larger strain hardening rate compared to the gradient-grained $\mathrm{Fe}$, as shown in Fig. 6(c). This enhanced strength and the reduced strain 

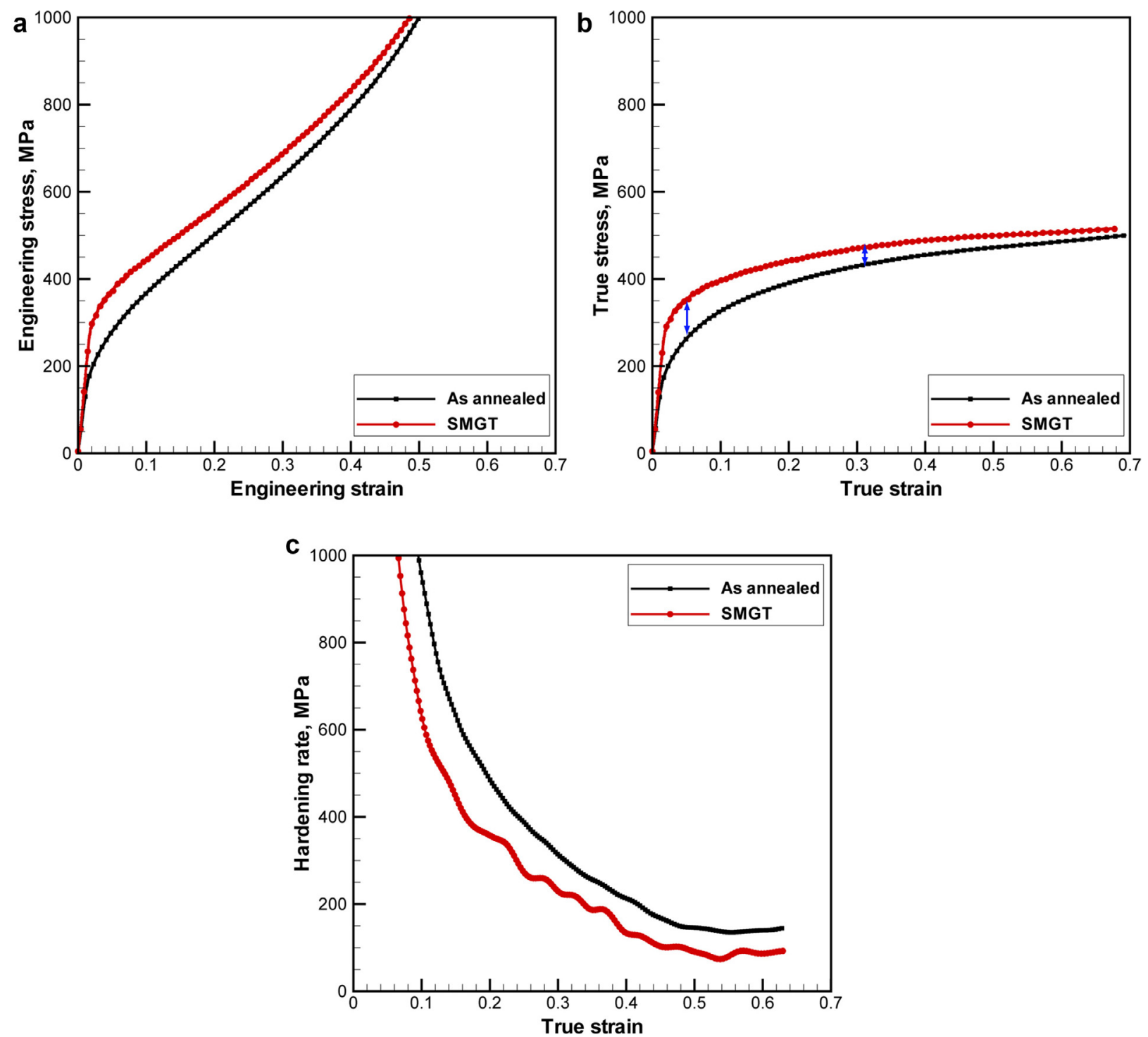

Fig. 6. (a) Engineering compressive stress-strain curves under quasi-static condition for as-annealed Fe and SMGT Fe; (b) true compressive stress-strain curves under quasi-static condition for as-annealed Fe and SMGT Fe; (c) strain hardening rate vs. true strain curves for as-annealed Fe and SMGT Fe.

hardening rate should be attributed to the refined strain size and the increased dislocation density at the surface layer of the gradient-grained Fe.

Fig. 7(a) and (b) shows typical true stress-true strain curves under dynamic compression at various strain rates ( $\sim 1500, \sim 6000, \sim 10,000 \mathrm{~s}^{-1}$ ) for the as-annealed Fe and the gradient-grained $\mathrm{Fe}$, respectively. In order to further show the strain hardening behaviors of the as-annealed $\mathrm{Fe}$ and the gradient-grained Fe under various strain rates, Ludwik-Hollomon equation was used to fit the true stresstrue strain curves as follows:

$\sigma=\sigma_{0}+K \varepsilon^{n}$

where $\sigma_{0}$ is the yield strength, $K$ is the strain hardening factor and $n$ is the strain hardening exponent which typically reflects the stain hardening ability. According to the Ludwik-Hollomon equation, double logarithm true stress- true strain curves at various strain rates are plotted in Fig. 8(a) and (b) in order to obtain the strain hardening exponents for the as-annealed Fe and the gradientgrained Fe. Typically, the CG metals exhibit relatively small yield stresses and apparent strain hardening behaviors even under dynamic compression, while much higher yield stresses but reduced strain hardening behaviors, sometimes even strain softening behaviors due to the thermal effect can be observed in UFG or NG metals under dynamic compression (Wei et al., 2004, 2006a; Mishra et al., 2008; Suo et al., 2011, 2013a). For the as-annealed Fe (Fig. 7(a)), apparent hardening behaviors are observed at all strain rates like other CG metals and the strain hardening ability (strain hardening exponent, $n$ ) decreases with increasing strain rate (Fig. 8(a)). Although the strain hardening exponent $(n)$ for the gradient-grained Fe is smaller than that of the CG Fe at the same strain rate, it is 

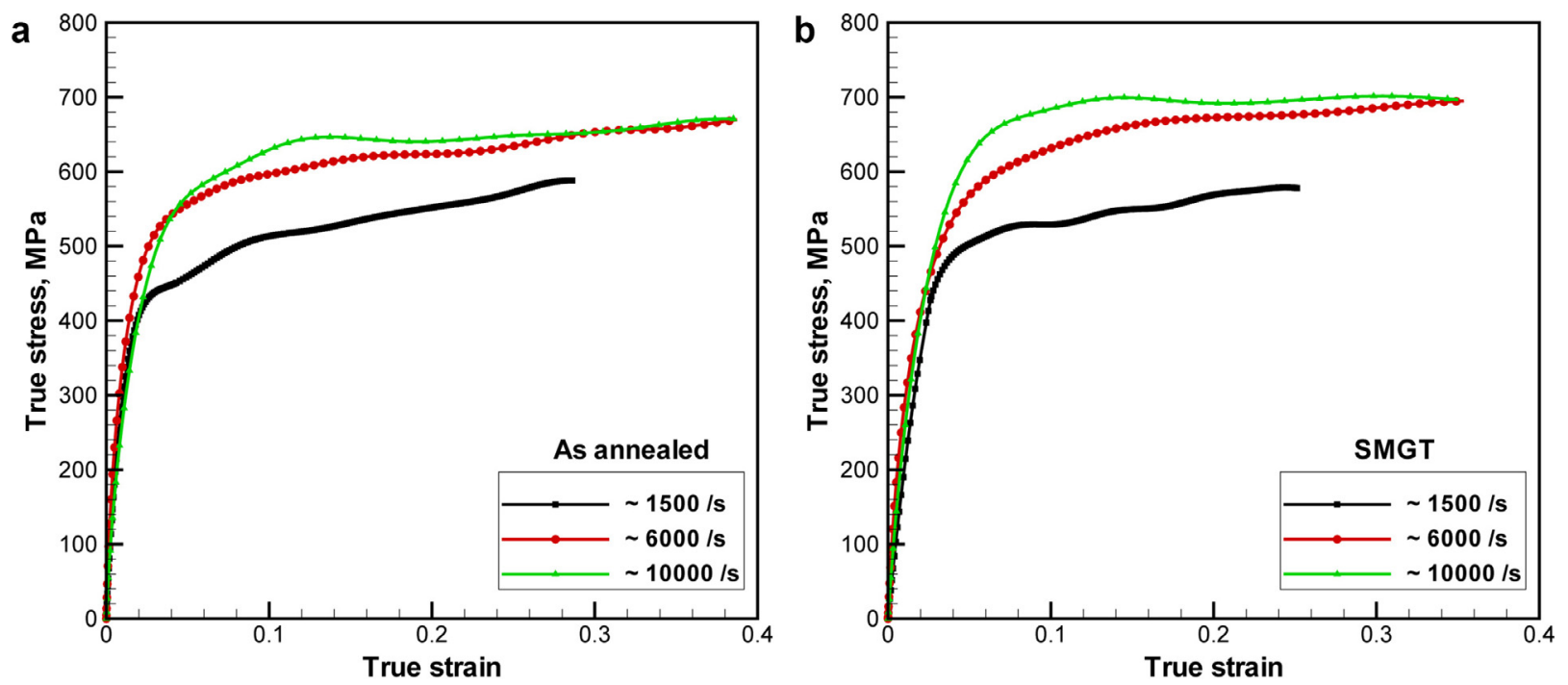

Fig. 7. True stress-true strain curves under dynamic compression: (a) as-annealed Fe; (b) SMGT Fe.
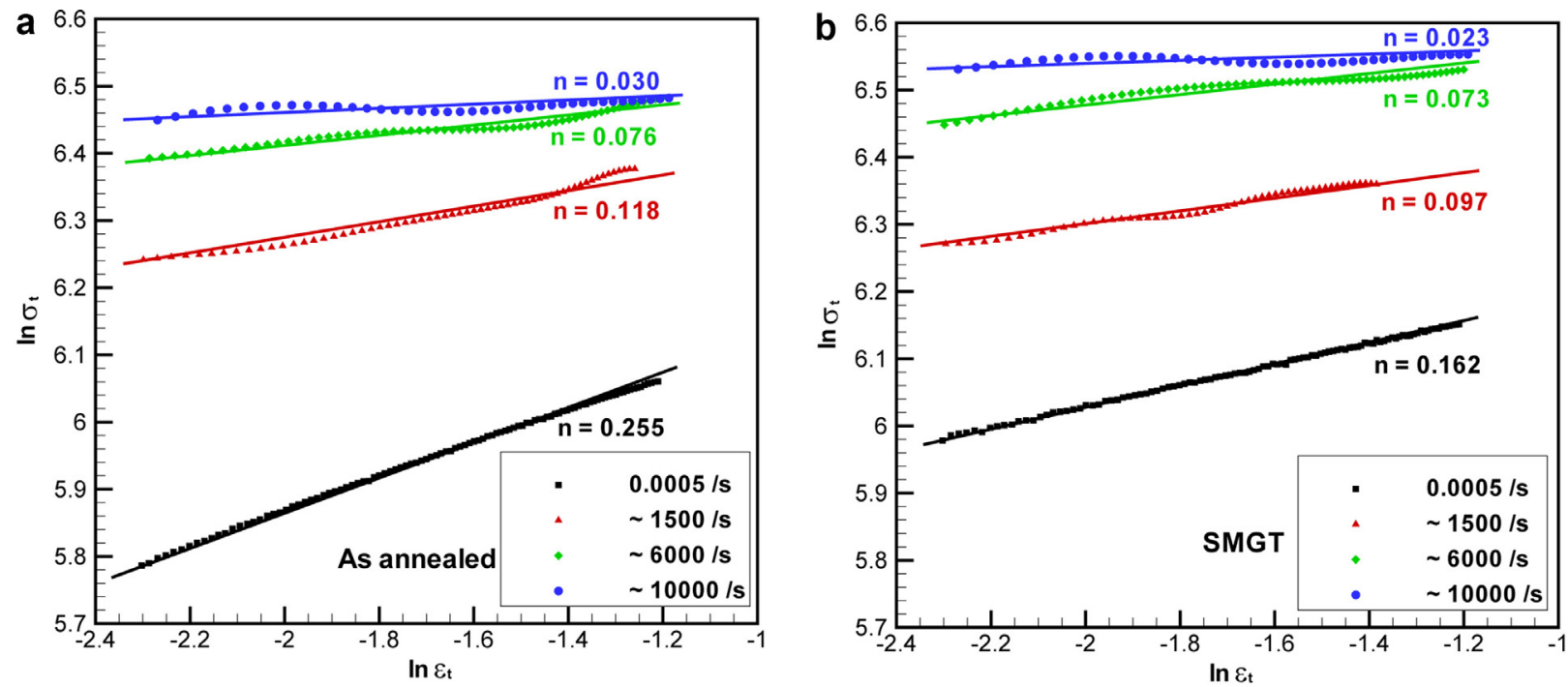

Fig. 8. True stress-true strain curves with double logarithm forms at various strain rates under compression: (a) as-annealed Fe; (b) SMGT Fe. The solid lines are the linear fit for the data.

interesting to note that the gradient-grained Fe still shows apparent strain hardening behaviors at all strain rates even when the thermal softening effect exists under dynamic compression.

The apparent hardening behavior under dynamic compression for the gradient-grained Fe raises a critical issue: where is the strain hardening generated? To answer this question, we measured the micro-hardness (Hv) along the depth from the treated surface for the gradient-grained Fe before and after dynamic compression. As shown in Fig. 9(a), the micro-hardness values increase at all depths after dynamic compression. The hardness increment $(\Delta H)$ along the depth after dynamic compression is also shown in Fig. 9(a), and $\Delta H$ is an indicator on the magnitude of hardening retained after unloading. It should be noted that the first indentation is at the depth of $10 \mu \mathrm{m}$ from the treated surface and the indentation size is about $7 \mu \mathrm{m}$, thus the hardness contribution for the first indentation should come from both the equiaxed ultrafine grains (with grain size of $\sim 100 \mathrm{~nm}$ ) and the elongated lamellar ultrafine grains (with grain size of $\sim 200 \mathrm{~nm}$ ). It is not surprised that strong hardness increment (about $60 \mathrm{Hv}$ ) occurs in the CG center since the coarse grains have the abilities for strain hardening. However, it is interesting to note that the surface layer with equiaxed/lamellar ultrafine grains (100-200 nm) also shows large hardness increment (about $60 \mathrm{Hv}$ ) after dynamic compression, which is completely different to the general observations that disappeared strain hardening behaviors, sometimes even strain softening behaviors should be observed in UFG or NG metals under dynamic compression (Wei et al., 2004, 2006a; Mishra et al., 2008; Suo et al., 2011, 2013a). The strain 
a

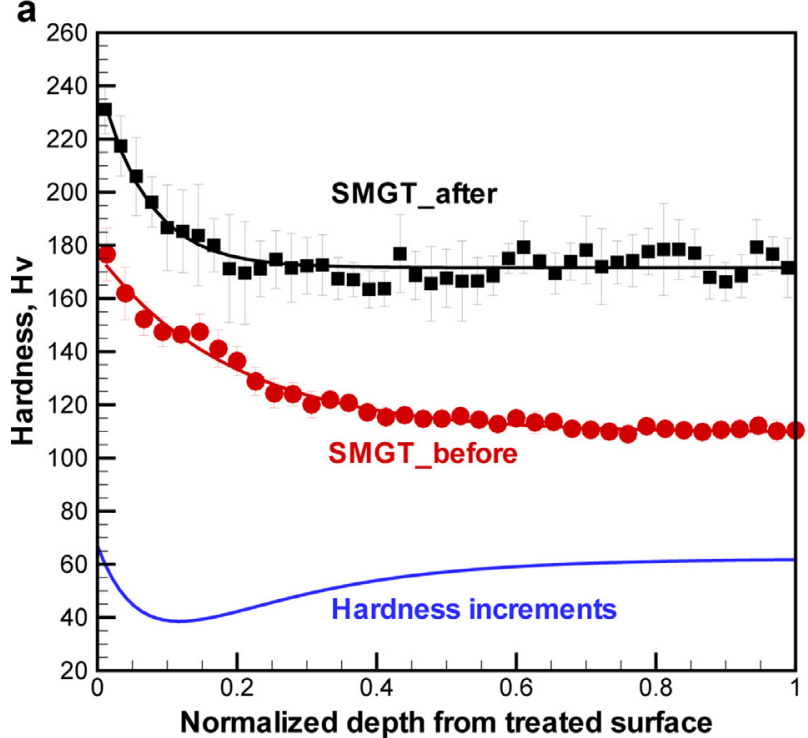

b

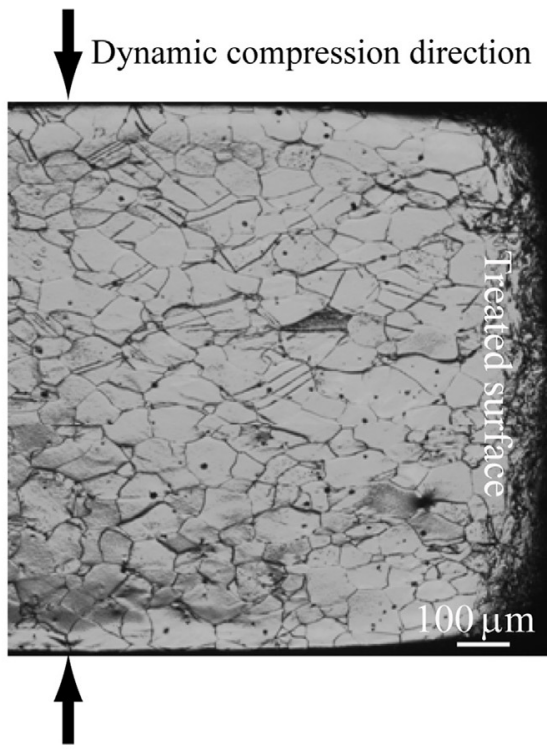

Fig. 9. (a) Vickers micro-hardness distributions for SMGT Fe before and after dynamic compression (strain rate of $\sim 1500 \mathrm{~s}^{-1}$ ), the symbols are the measured data points and the solid lines are the fitting curves; (b) OM of the gradient-grained Fe after dynamic compression.

softening behaviors in UFG or NG metals under dynamic compression were explained due to the thermal softening and the formation of adiabatic shear band (Wei et al., 2004, 2006a; Mishra et al., 2008), however this strain localization trend for the surface layer of the gradient-grained Fe would be suppressed by the CG center and the mechanical constraint would be generated between different layers in the gradient-grained Fe. Our optical micrograph after dynamic compression confirmed this suggestion and no adiabatic shear band was observed for the surface layer of the gradient-grained $\mathrm{Fe}$ (as shown in Fig. 9(b)). Thus, based on these observations mentioned above, the apparent strain hardening behaviors under dynamic compression for the gradient-grained Fe could be understood as three-fold: (1) first, the CG center provides the important part of the strain hardening ability for the gradient-grained Fe; (2) second, the constraint and mechanical incompatibility between different layers should produce extra hardening for the surface gradient layers due to back stress hardening (Feaugas, 1999; Elliot et al., 2004; Wu et al., 2015) and the GNDs induced by the grain size gradient (Gao et al., 1999; Wu et al., 2014a,b); (3) the restrain of dynamic recovery of dislocations at high strain rates (Suo et al., 2011) may also contribute to the strain hardening under dynamic conditions.

Due to the suppression of adiabatic shear band by the CG center, the dynamic homogeneous compression strain can be much improved in the gradient-grained Fe. The energy absorption under dynamic compression up to a specific strain can be calculated as the areas under the true stress-strain curves in Fig. 7(a). Driven by the need to retain impact toughness while reaping the strengthening benefits from the gradient-grained structure, the gradientgrained structure reported here can be used to design components of energy absorbers for the automotive industry, which is illustrated in Fig. 10. In Fig. 10, the energy ab-

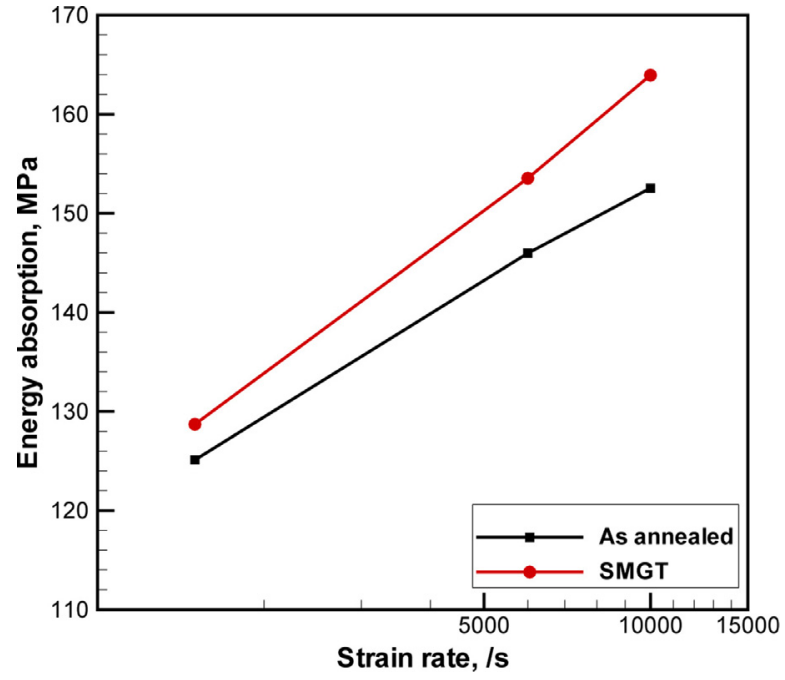

Fig. 10. Energy absorption up to a true strain of 0.25 vs. strain rate: (a) as-annealed Fe; (b) SMGT Fe.

sorption under dynamic compression is plotted against the strain rate for the as-annealed Fe and the gradient-grained Fe. As indicated, besides with higher yield strength, the gradient-grained Fe also has higher energy absorption under dynamic compression at all strain rates, when compared to the as-annealed Fe.

Fig. 11(a)-(c) shows the flow stress as a function of the strain rate with double logarithmic coordinates for both the as-annealed $\mathrm{Fe}$ and the gradient-grained $\mathrm{Fe}$ at fixed true strains of $10 \%, 15 \%$ and $20 \%$, respectively. At all fixed true strains, the flow stress of the gradient-grained $\mathrm{Fe}$ is clearly observed to increase slightly faster than that of the as-annealed Fe with increasing strain rate. Both the 

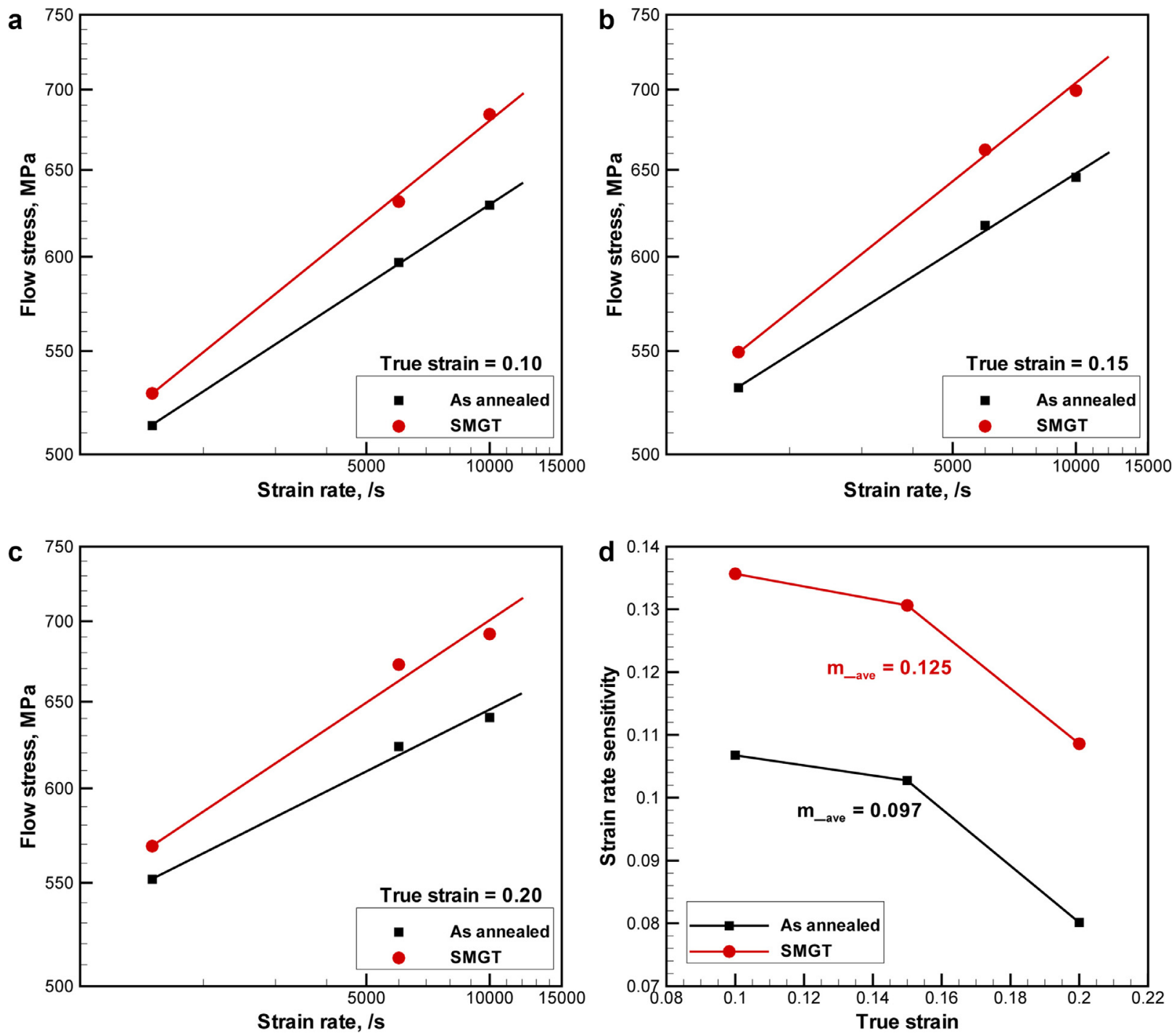

Fig. 11. Flow stress vs. strain rate for as-annealed Fe and SMGT Fe: (a) at 10\% true strain; (b) at 15\% true strain; (c) at 20\% true strain. Double logarithm coordinates are used in Fig. 11(a)-(c), and the solid lines are the linear fit for the data. (d) Strain rate sensitivity vs. true strain for as-annealed Fe and SMGT Fe.

as-annealed $\mathrm{Fe}$ and the gradient-grained $\mathrm{Fe}$ show positive SRS under dynamic compression.

In general, the plastic deformation of metals and alloys depends on the strain, the strain rate and the deformation temperature, thus the flow stress can be written as:

$\sigma=f(\varepsilon, \dot{\varepsilon}, T)$

where $\varepsilon$ is the true strain, $\dot{\varepsilon}$ is the true strain rate, and $T$ is the environment temperature associated with the loading. Based on Eq. (5), the SRS of flow stress can be defined as:

$m=\left(\frac{\partial \ln \sigma}{\partial \ln \dot{\varepsilon}}\right)_{\varepsilon, T}$

In practice, the SRS is often calculated at certain fixed temperature and at several fixed strains. Under quasi-static conditions, the SRS is generally determined using the strain rate jump tests or stress relaxation tests. However, these two experiments are hard to employ under dynamic conditions. Alternatively, the SRS can also be obtained approximately by using the true stress-strain curves with double logarithmic form at a fixed true strain, where the SRS is derived as the slope of a linear regression fit for the curves. By using this method, the dynamic SRS as a function of the true strain is plotted in Fig. 11(d) for both the as-annealed Fe and the gradient-grained Fe. The dynamic SRS is observed to decrease from 0.107 to 0.08 when the true strain is changed from $10 \%$ to $20 \%$ for the as-annealed Fe, and the average dynamic SRS is about 0.097. While, the dynamic SRS decreases from 0.136 to 0.109 when the true strain is changed from $10 \%$ to $20 \%$ for the gradient-grained $\mathrm{Fe}$, and the average dynamic SRS is about 0.125. It is interesting to note that the dynamic SRS in our experiments are much higher than those from the literature $(<0.04)$ (Wei, 2007). 
The SRS values from previous research for UFG or NG metals (Wei, 2007) are mostly obtained from quasi-static loading (nanoindentation, strain rate jump tests or stress relaxation tests), and the SRS values under quasi-static conditions are typically lower than those under dynamic conditions due to possibly different deformation mechanisms (Meyers, 1994; Suo et al., 2013b). According to the previous research (Wei, 2007), the SRS of BCC metal is observed generally to decrease with the reduction of grain size. The gradient-grained Fe has smaller average grain size and should have smaller SRS according to previous work (Wei, 2007), when compared to the as-annealed Fe. However, the results in the present study show different trends for SRS of BCC metals with reduction of grain size compared to previous work (Wei, 2007), and the possible reasons will be two-fold: (1) first, the SRS values are obtained from different strain rate regimes for our data and the data from previous work (Wei, 2007); (2) more importantly, the other possible reason is the reduced activation volume by the gradient structure, and will be presented in details in the following discussion.

Base on the concept of thermal-activation theory, the apparent activation volume $\left(V^{*}\right)$ can be estimated

$V^{*}=\frac{\sqrt{3} k T}{\sigma m}$

where $k$ is Boltzmann's constant, $T$ is the absolute temperature associated with loading, $m$ is the SRS, and $\sigma$ is the flow stress.

Thus, the SRS can written as

$m=\frac{\sqrt{3} k T}{\sigma V^{*}}$

Typically, the physical activation volume is highly dependent on the dislocation density. The higher the dislocation density, the lower is the activation volume (Wei, 2007).

According to the previous research (Wei, 2007), the activation volume decreases to nearly a constant when the stress is increased to a moderate level for BCC metals, however the stress is still following the Hall-Petch relation when the grain size is refined. Thus, the SRS should decrease with reduced grain size for BCC metals based on Eq. (8).

However, the dislocation density could be further increased by the additional dislocations from the GNDs associated with the back stress hardening (Feaugas, 1999; Elliot et al., 2004; Wu et al., 2015) and the grain size gradient (Gao et al., 1999; Wu et al., 2014a,b) when the constraint and mechanical incompatibility between different layers are produced in the gradient-grained structure. This additional increase in dislocation density could possibly result in lower activation volume, thus larger SRS $(m)$, which may be the reason why the gradient-grained Fe has slightly higher SRS even it has smaller average grain size compared to the as-annealed Fe.

\section{Conclusions}

In the present work, gradient-grained Fe was synthesized using SMGT, and then the microstructure after SMGT was examined by OM, EBSD, TEM and micro-hardness measurements. The strain hardening behaviors and strain rate sensitivity of the as-annealed Fe and the gradientgrained Fe under compression were then studied over a wide range of strain rates (from $5 \times 10^{-4}$ to $10^{4} \mathrm{~s}^{-1}$ ). The main findings are summarized as follows:

(1) From the treated surface to the center, equiaxed ultrafine grains, elongated lamellar ultrafine grains, sub-grains and full-developed dislocations walls, non-fully-developed dislocation cells, and deformed coarse grains are sequentially observed for the gradient-grained Fe. The grain/cell size increases while the measured micro-hardness decreases along the depth for the gradient-grained Fe.

(2) Although the strain hardening exponent $(n)$ for the gradient-grained $\mathrm{Fe}$ is smaller than that of the asannealed $\mathrm{Fe}$ at the same strain rate, the gradientgrained structure still shows apparent strain hardening behaviors at all strain rates up to $10^{4} \mathrm{~s}^{-1}$. Based on the micro-hardness measurements before and after dynamic compression, this apparent hardening behavior could be attributed to both hardening from the CG center and the surface gradient layers. The constraint and mechanical incompatibility between different layers in gradient-grained structure should be produced when the strain localization trend for the surface layer of the gradientgrained Fe is suppressed by the CG center. The constraint and mechanical incompatibility between different layers in gradient-grained structure should produce extra hardening for the surface gradient layers due to back stress hardening (Feaugas, 1999; Elliot et al., 2004; Wu et al., 2015).

(3) The dynamic SRS of the gradient-grained $\mathrm{Fe}$ is slightly larger than that of the as-annealed Fe, which is controversial to the general observations from previous work (Wei, 2007) that SRS should decrease with reduction of grain size for BCC metals. The GNDs associated with the back stress hardening and the grain size gradient can result in additional increase of dislocation density, which may be the reason why the gradient-grained Fe has slightly higher SRS even it has smaller average grain size compared to the as-annealed Fe.

The present results should advance our understanding of compression behaviors for the gradient-grained structure under extreme conditions. The gradient structure should also have promising applications for structural materials and energy absorption due to the high strength, the apparent strain hardening behaviors at all strain rates up to $10^{4} \mathrm{~s}^{-1}$ and the enhanced dynamic strain rate sensitivity.

\section{Acknowledgments}

The authors would like to acknowledge the financial support from the National Key Basic Research Program of China under grants no. 2012CB932203 and no. 2012CB937500; and NSFC under grants no. 11222224, no. 
11472286, 11072243, 11572328, and no. 11021262. The authors would like to thank Dr. Husheng Zhang for helping to conduct the dynamic compression experiments.

\section{References}

Chen, A.Y., Li, D.F., Zhang, J.B., Song, H.W., Lu, J., 2008. Make nanostructured metal exceptionally tough by introducing non-localized fracture behaviors. Scr. Mater 59, 579-581.

Chen, D., Kuo, J.C., Wu, W.T., 2011a. Effect of microscopic parameters on EBSD spatial resolution. Ultramicroscopy 111, 1488-1494.

Chen, Y.J., Li, Y.J., Walmsley, J.C., Dumoulin, S., Gireesh, S.S., Armada, S. 2011b. Quantitative analysis of grain refinement in titanium during equal channel angular pressing. Scr. Mater. 64, 904-907.

Conrad, H., 2004. Grain-size dependence of the flow stress of $\mathrm{Cu}$ from millimeters to nanometers. Metall. Mater. Trans. A 35, 2681-2695.

Dao, M., Lu, L, Asaro, R.J., De, Hosson, J.T.M., Ma, E., 2007. Toward a quantitative understanding of mechanical behavior of nanocrystalline metals. Acta Mater 55, 4041-4065.

Elliot, R.A., Orowan, E., Udoguchi, T., Argon, A.S., 2004. Absence of yield points in iron on strain reversal after aging, and the Bauschinger overshoot. Mech. Mater. 36, 1143-1153.

Fang, T.H., Li, W.L., Tao, N.R., Lu, K., 2011. Revealing extraordinary intrinsic tensile plasticity in gradient nano-grained copper. Science 331, 15871590.

Feaugas, X., 1999. On the origin of the tensile flow stress in the stainless steel AISI $316 \mathrm{~L}$ at $300 \mathrm{~K}$ : back stress and effective stress. Acta Mater 47, 3617-3632.

Gao, H., Huang, Y., Nix, W.D., Hutchinson, J.W., 1999. Mechanisms-based strain gradient plasticity. I. Theory. J. Mech. Phys. Solids 47, 12391263.

Giga, A., Kimoto, Y., Takigawa, Y., Higashi, K., 2006. Demonstration of an inverse Hall-Petch relationship in electrodeposited nanocrystalline Ni-W alloys through tensile testing. Scr. Mater. 55, 143-146.

Han, B.Q., Lavernia, E.J., Mohamed, A., 2003. Mechanical properties of iron processed by severe plastic deformation. Metall. Mater. Trans. A 34A 71-83.

Han, B.Q., Lavernia, E.J., Mohamed, A., 2004. Dislocation structure and deformation in iron processed by Equal-Channel-Angular pressing. Metall. Mater. Trans. A 35A, 1343-1350.

Hazra, S.S., Pereloma, E.V., Gazder, A.A., 2011. Microstructure and mechanical properties after annealing of equal-channel angular pressed interstitial-free steel. Acta Mater. 59, 4015-4029.

Jia, D., Ramesh, K.T., Ma, E., 1999. Failure mode and dynamic of nanophase iron under compression. Scr. Mater. 42, 73-78.

Li, W.L., Tao, N.R., Lu, K., 2008. Fabrication of a gradient nano-microstructured surface layer on bulk copper by means of a surface mechanical grinding treatment. Scr. Mater. 59, 546-549.

Liddicoat, P.V., Liao, X.Z., Zhao, Y.H., Zhu, Y.T., Murashkin, M.Y., Lavernia, E.J., Valiev, R.Z., Ringer, S.P., 2010. Nanostructural hierarchy increases the strength of aluminium alloys. Nat. Commun. 1, Article no. 63.

Liu, G., Zhang, G.J., Jiang, F., Ding, X.D., Sun, Y.J., Sun, J., Ma, E. 2013. Nanostructured high-strength molybdenum alloys with unprecedented tensile ductility. Nat. Mater 12, 344-350.

Lu, K., Lu, J., 2004. Nanostructured surface layer on metallic materials induced by surface mechanical attrition treatment. Mater. Sci. Eng. A 375-377, 38-45.

Lu, K., Lu, L., Suresh, S., 2009. Strengthening materials by engineering coherent internal boundaries at the nanoscale. Science 324, 349-352.

Malow, T.R., Koch, C.C., 1998. Mechanical properties, ductility, and grain size of nanocrystalline iron produced by mechanical attrition. Metall. Mater. Trans. A 29A, 2285-2295.

Meyers, M.A., 1994. Dynamic Behavior of Materials. Wiley-Interscience, New York, USA, pp. 323-326.

Meyers, M.A., Mishra, A., Benson, D.J., 2006. Mechanical properties of nanocrystalline materials. Prog. Mater. Sci. 51, 427-556.

Mishra, A., Martin, M., Thadhani, N.N., Kad, B.K., Kenik, E.A., Meyers, M.A., 2008. High-strain rate response of ultra-fine-grained copper. Acta Mater. 56, 2770-2783.

Nemat-Nasser, S., Guo, W.G., 2003. Thermomechanical response of DH36 structural steel over a wide range of strain rate and temperatures. Mech. Mater. 35, 1023-1047.
Song, B., Chen, W., Antoun, B.R., 2007. Determination of early flow stress for ductile specimens at high strain rates by using a SHPB. Exp. Mech. 47, 671-679.

Subhash, G., 1995. The constitutive behavior of refractory-metals as a function of strain-rate. JOM 5, 55-58.

Subhash, G., Ravichandran, G., Pletka, B.J., 1997. Plastic deformation of hafnium under uniaxial compression. Metall. Mater. Trans. A 28A, 1479-1487

Sun, J.L., Trimby, P.W., Yan, F.K., Liao, X.Z., Tao, N.R., Wang, J.T., 2014. Shear banding in commercial pure titanium deformed by dynamic compression. Acta Mater 79, 47-58.

Sunny, G., Yuan, F.P., Lewandowski, J.J., Prakash, V., 2009. Design of inserts for split-Hopkinson pressure bar testing of low strain-to-failure materials. Exp. Mech. 49, 479-490.

Suo, T., Li, Y.L., Xie, K., Zhao, F., Zhang, K.S., Deng, Q., 2011. Experimental investigation on strain rate sensitivity of ultra-fine grained copper at elevated temperatures. Mech. Mater. 43, 111-118.

Suo, T., Li, Y.L., Zhao, F., Fan, X.L., Guo, W.G., 2013a. Compressive behavior and rate-controlling mechanisms of ultrafine grained copper over wide temperature and strain rate ranges. Mech. Mater. 61, 1-10.

Suo, T., Chen, Y.Z., Li, Y.L., Wang, C.X., Fan, X.L., 2013b. Strain rate sensitivity and deformation kinetics of ECAPed aluminum over a wide range of strain rates. Mater. Sci. Eng. A 560, 545-551.

Suresh, S., 2011. Graded materials for resistance to contact deformation and damage. Science 292, 2447-2451.

Tsuji, N., Ito, Y., Saito, Y., Minamino, Y., 2002. Strength and ductility of ultrafine grained aluminum and iron produced by ARB and annealing. Scr. Mater 47, 893-899.

Valiev, R., 2004. Nanostructuring of metals by severe plastic deformation for advanced properties. Nat. Mater 3, 511-516.

Wang, Y.M., Chen, M.W., Zhou, F.H., Ma, E., 2002. High tensile ductility in a nanostructured metal. Nature 419, 912-915.

Wei, Q., Kecskes, L., Jiao, T., Hartwig, K.T., Ramesh, K.T., Ma, E., 2004. Adiabatic shear banding in ultrafine-grained Fe processed by severe plastic deformation. Acta Mater. 52, 1859-1869.

Wei., Q., Jiao, T., Ramesh, K.T., Ma, E., Kecskes, L.J., Magness, L., Dowding, R., Kazykhanov, V.U., Valiev, R.Z., 2006a. Mechanical behavior and dynamic failure of high-strength ultrafine grained tungsten under uniaxial compression. Acta Mater. 54, 77-87.

Wei, Q., Zhang, H.T., Schuster, B.E., Ramesh, K.T., Valiev, R.Z., Kecskes, L.J., Dowding, R.J., Magness, L., Cho, K., 2006b. Microstructure and mechanical properties of super-strong nanocrystalline tungsten processed by high-pressure torsion. Acta Mater 54, 4079-4089.

Wei, Q., 2007. Strain rate effects in the ultrafine grain and nanocrystalline regimes e influence on some constitutive responses. J. Mater. Sci. 42, 1709-1727.

Wei, Y.J., Li, Y.Q., Zhu, L.C., Liu, Y., Lei, X.Q., Wang, G., Wu, Y.X., Mi, Z.L., Liu, J.B., Wang, H.T., Gao, H.J., 2014. Evading the strength-ductility trade-off dilemma in steel through gradient hierarchical nanotwins. Nat. Commun 5, Article no. 3580

Wu, X.L., Jiang, P., Chen, L., Yuan, F.P., Zhu, Y.T., 2014a. Extraordinary strain hardening by gradient structure. Proc. Natl. Acad. Sci. U.S.A. 111, 71977201.

Wu, X.L., Jiang, P., Chen, L., Zhang, J.F., Yuan, F.P., Zhu, Y.T., 2014b. Synergetic strengthening by gradient structure. Mater. Res. Lett. 2, 185191.

Wu, X.L., Yang, M.X., Yuan, F.P., Wu, G.L., Wei, Y.J., Huang, X.X., Zhu, Y.T., 2015. Heterogeneous lamellar structure unites ultrafine-grain strength with coarse-grain ductility. Proc. Natl. Acad. Sci. USA 112, 1450114505.

Yu, X., Li, Y.L., Wei, Q.M., Guo, Y.Z., Suo, T., Zhao, F., 2015. Microstructure and mechanical behavior of ECAP processed AZ31B over a wide range of loading rates under compression and tension. Mech. Mater. 86, 5570.

Yuan, F.P., Bian, X.D., Jiang, P., Yang, M.X., Wu, X.L., 2015. Dynamic shear response and evolution mechanisms of adiabatic shear band in an ultrafine-grained austenite-ferrite duplex steel. Mech. Mater. 89, 4758.

Zener, C., Hollomon, J.H., 1944. Effect of strain rate upon plastic flow of steel. J Appl. Phys. 15, 22-32.

Zhu, Y.T., Liao, X.Z., 2004. Nanostructured metals - retaining ductility. Nat. Mater 3, 351-352. 